\title{
Distributed Space-Frequency Coding over Broadband Relay Channels
}

\author{
Karim G. Seddik and K. J. Ray Liu, Fellow, IEEE
}

\begin{abstract}
Designing diversity achieving schemes over the wireless broadband fading relay channels is crucial to achieve higher diversity gains. These gains are achieved by exploiting the multipath (frequency) and cooperative diversities to combat the fading nature of wireless channels. The challenge is how to design spacefrequency codes, distributed among randomly located nodes that can exploit the frequency diversity of the wireless broadband channels. In this paper, the design of distributed space-frequency codes (DSFCs) for wireless relay networks is considered. The proposed DSFCs are designed to achieve the frequency and cooperative diversities of the wireless relay channels. The use of DSFCs with the decode-and-forward (DAF) and amplify-andforward (AAF) protocols is considered. The code design criteria to achieve full diversity, based on the pairwise error probability (PEP) analysis, are derived. For DSFC with the DAF protocol, a two-stage coding scheme, with source node coding and relay nodes coding, is proposed. We derive sufficient conditions for the proposed code structures at the source and relay nodes to achieve full diversity of order $N L$, where $N$ is the number of relay nodes and $L$ is the number of paths per channel. For the case of DSFC with the AAF protocol, a structure for distributed space-frequency coding is proposed.
\end{abstract}

Index Terms-Amplify-and-forward, broadband channels, decode-and-forward, relay channels, space-frequency coding.

\section{INTRODUCTION}

$\mathbf{T}$ HE advent of future broadband wireless services, which require high signal quality and high data rates, has focused attention on the study of wireless channels. Wireless resources such as the bandwidth are scarce, and it is difficult to meet the high data rate requirement unless efficient techniques are employed. Also, wireless channels have many impairments such as fading, shadowing, and multiuser interference, which can highly degrade the system performance. Spatial diversity has proved to be a prime candidate for achieving the signal quality and high data rate promised by the future broadband wireless services. Spatial diversity is also of special interest as it does not increase the overhead in the system in terms of the bandwidth and the delay.

The seminal work in [1] has shown the increased capacity of the wireless Multiple-Input Multiple-Output (MIMO) channels, which are constructed through the use of multiple transmit and/or multiple receive antennas. In wireless applications, it is affordable to have multiple antennas at the base station,

Manuscript received October 29, 2007; accepted February 7, 2008. The associate editor coordinating the review of this paper and approving it for publication was X.-G. Xia.

K. G. Seddik is with the Department of Electrical Engineering, Alexandria University, Alexandria 21544 Egypt (e-mail: kseddik@ieee.org).

K. J. R. Liu is with the Department of Electrical and Computer Engineering, and the Institute for Systems Research, University of Maryland, College Park, MD 20742 (e-mail: kjrliu@umd.edu).

Digital Object Identifier 10.1109/T-WC.2008.071205 but it is difficult to equip small mobile units with more than one antenna due to their space constraints. This led to what is known as cooperative diversity, in which the nodes try to form a virtual multiple element transmit antenna. Cooperative diversity is achieved through relay nodes that help the source node by forwarding its information.

The classical relay channel model based on additive white Gaussian noise (AWGN) channels was presented in [2]. Lately, the study of cooperative diversity achieving techniques has gained significant interest. In [3], different protocols were proposed to achieve spatial diversity through node cooperation. Among them are the decode-and-forward (DAF) and amplifyand-forward (AAF) protocols. In the decode-and-forward protocol with one relay node, the relay node decodes the source node symbol before re-transmitting the source node data to the destination node. In the amplify-and-forward protocol with one relay node, the relay node amplifies the received source node signal before re-transmission to the destination node.

The problem with the previous protocols is the data rate loss as the number of relays increases, since relays are assumed to be assigned orthogonal channels (for example, different time slots in TDMA systems). This leads to the use of what is known as distributed space-time coding (DSTC), where relay nodes are allowed to simultaneously transmit over the same channel by emulating a space-time code [4]. DSTCs are designed to achieve diversity over flat, frequency nonselective, fading channels. In DSTC, relay nodes try to emulate a multiple antenna transmitter, where relay nodes simultaneously transmit in the cooperation phase. Several works have considered the design of distributed space-time codes for the wireless relay channels [5], [6], [7].

For the case of broadband multipath fading channels the design of distributed space-frequency codes (DSFCs) is needed to exploit the frequency diversity of the channel. The presence of multipaths in broadband channels provides another means for achieving diversity across the frequency axis. Exploiting the frequency axis diversity can highly improve the system performance by achieving higher diversity orders. The design of space-frequency codes over MIMO channels was considered, for example, in [8] and references therein. The main problem for the wireless relay network is how to design space-frequency codes distributed among spatially separated relay nodes while guaranteeing to achieve full diversity at the destination node. The spatial separation of the relay nodes presents other challenges for the design of DSFCs such as time synchronization. We will address these code design aspects in our proposed DSFC structures.

A design of DSFCs was considered in [9], where a system 
that employs the DAF protocol was used. It was assumed that all of the relay nodes will always decode correctly, which is not always true especially over wireless fading channels. Such proposed DSFCs mitigate synchronization errors due to the timing mismatches of relay nodes and propagation delays by employing OFDM transmission. The presence of the cyclic prefix in OFDM modulation can mitigate synchronization errors of relay nodes ${ }^{1}$. Hence, perfect synchronization, which means perfect timings of relay nodes and propagation delays synchronization, is not necessary, which greatly simplifies the system design.

In this paper, we consider the design of distributed spacefrequency codes (DSFCs) that can exploit the multipath nature of broadband wireless channels. We consider the use of DAF and AAF protocols, which are the most commonly used cooperation protocols. First, the design of DSFC with the DAF protocol is considered. Our system model is different from the model considered in [9], where relays are assumed to always decode correctly. We consider a system where relay nodes can decode the information from the source node erroneously. The system will have two phases as follows. In the first phase, the source broadcasts its information to the relay nodes. In phase 2 , the relays that have correctly decoded the source data will help the source forwarding its information by emulating a multiple antenna transmitter while relays that have decoded erroneously remain idle.

A two-stage coding, namely, the source node coding and the relay node coding is proposed to design the DSFCs. Sufficient conditions for the proposed two-stage DSFC structure to achieve full diversity of order $N L$, where $N$ is the number of cooperating relay nodes and $L$ is the number of paths per channel, are derived. Second, the design of DSFC with the AAF protocol is considered. In the AAF protocol, relay nodes can only apply simple operations on the received source signal such as normalizing and amplifying the signal. A structure for DSFC with the AAF protocol is proposed. The proposed code structure is showed to achieve full diversity of order $N L$ for some special cases.

The rest of the paper is organized as follows. In Section II, the system model and performance analysis for DSFCs with the DAF protocol are presented. In Section III, the system model and performance analysis for DSFCs with the AAF protocol are presented. Section IV presents a construction for the proposed DSFCs and a discussion about other design aspects of the proposed code structures. In Section V, simulation results are presented. Finally, Section VI concludes the paper.

\section{DSFC WITH THE DAF PROTOCOL}

In this section, the design and performance analysis for DSFCs with the DAF protocol are presented. A two-stage structure is proposed for the DSFCs with the DAF protocol. Sufficient conditions for the proposed code structure to achieve full diversity are derived.

\section{A. System Model}

In this section, the system model for the DSFCs with the DAF protocol is presented. In this paper $\lfloor x\rfloor$ denotes the

\footnotetext{
${ }^{1}$ This follows directly due to the use of OFDM transmission and the same applies for our proposed DSFCs.
}

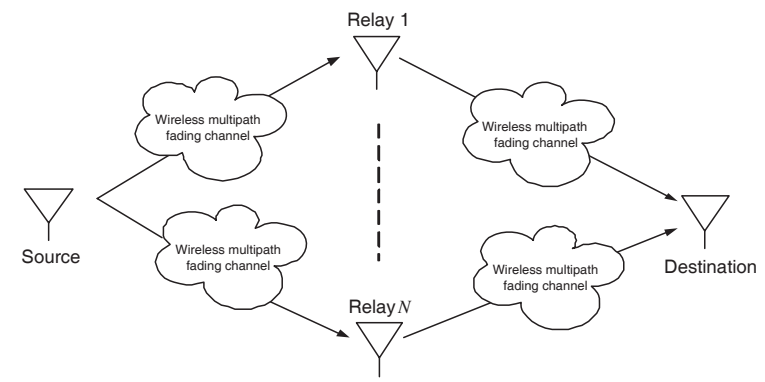

Fig. 1. Simplified system model for the distributed space-frequency codes.

largest integer that is less that $x$. $\operatorname{diag}(\mathbf{y})$, where $\mathbf{y}$ is a $T \times 1$ vector, is the $T \times T$ diagonal matrix with the elements of $\mathbf{y}$ on its diagonal. $\mathbf{A} \otimes \mathbf{B}$ denotes the tensor product of the two matrices $\mathbf{A}$ and $\mathbf{B}$. $\|\mathbf{A}\|_{F}^{2}$ of the $m \times n$ matrix $\mathbf{A}$ is the Frobenius norm of the matrix defined as $\|\mathbf{A}\|_{F}^{2}=$ $\sum_{i=1}^{m} \sum_{j=1}^{n}|\mathbf{A}(i, j)|^{2}=\mathcal{T} \mathcal{R}\left(\mathbf{A} \mathbf{A}^{\mathcal{H}}\right)=\mathcal{T} \mathcal{R}\left(\mathbf{A}^{\mathcal{H}} \mathbf{A}\right)$ where $\mathcal{T} \mathcal{R}(\cdot)$ is the trace of a matrix.

Without loss of generality, we assume a two-hop relay channel model, where there is no direct link from the source node to the destination node. The case when a direct link exists between the source node and the destination node will be discussed in Section IV. A schematic system model is depicted in Fig. 1. The system is based on OFDM modulation with $K$ subcarriers. The channel between the source node and the $n$-th relay node is modeled as a multipath fading channel with $L$ paths as

$$
h_{s, r_{n}}(\tau)=\sum_{l=1}^{L} \alpha_{s, r_{n}}(l) \delta\left(\tau-\tau_{l}\right),
$$

where $\tau_{l}$ is the delay of the $l$-th path, $\delta(\cdot)$ is the Dirac delta function, and $\alpha_{s, r_{n}}(l)$ is the complex amplitude of the $l$-th path. The $\alpha_{s, r_{n}}(l)$ 's are modeled as zero-mean complex Gaussian random variables with variance $E\left[\left|\alpha_{s, r_{n}}(l)\right|^{2}\right]=\sigma^{2}(l)$, where we assume symmetry between the relay nodes for simplicity of presentation; the analysis can be easily extended to the asymmetric case. The channels are normalized such that the channel variance $\sum_{l=1}^{L} \sigma^{2}(l)=1$. A cyclic prefix is introduced to convert the multipath frequency-selective fading channels to flat fading subchannels on the subcarriers.

The system has two phases as follows. In phase 1, the source node broadcasts the information to the $N$ relays. The received signal in the frequency domain on the $k$-th subcarrier at the $n$-th relay node is given by

$$
\begin{gathered}
y_{s, r_{n}}(k)=\sqrt{P_{s}} H_{s, r_{n}}(k) s(k)+\eta_{s, r_{n}}(k), \\
k=1, \cdots, K ; \quad n=1, \cdots, N,
\end{gathered}
$$

where $P_{s}$ is the transmitted source node power, $H_{s, r_{n}}(k)$ is the channel attenuation of the source node to the $n$-th relay node channel on the $k$-th subcarrier, $s(k)$ is the transmitted source node symbol on the $k$-th subcarrier with $E\left\{|s(k)|^{2}\right\}=1$, and $\eta_{s, r_{n}}(k)$ is the $n$-th relay node additive white Gaussian noise on the $k$-th subcarrier that is modeled as zero-mean circularly symmetric complex Gaussian random variable with variance $N_{0} / 2$ per dimension. The subcarrier noise terms are statistically independent assuming that the time-domain 
noise samples are statistically independent and identically distributed $^{2}$. In (2), $H_{s, r_{n}}(k)$ is given by

$$
H_{s, r_{n}}(k)=\sum_{l=1}^{L} \alpha_{s, r_{n}}(l) e^{-j 2 \pi(k-1) \Delta f \tau_{l}}, k=1, \cdots, K,
$$

where $\Delta f=1 / T$ is the subcarrier frequency separation and $T$ is the OFDM symbol duration. We assume perfect channel state information at any receiving node but no channel information at transmitting nodes.

In phase 2, relays that have decoded correctly in phase 1 will forward the source node information. Each relay is assumed to be able to decide whether it has decoded correctly or not. This can be achieved through the use of error detecting codes such as the Cyclic Redundancy codes (CRC) [10], [11].

The transmitted $K \times N$ space-frequency (SF) codeword from the relay nodes is given by ${ }^{3}$

$$
\mathbf{C}_{r}=\left(\begin{array}{cccc}
C_{r}(1,1) & C_{r}(1,2) & \cdots & C_{r}(1, N) \\
C_{r}(2,1) & C_{r}(2,2) & \cdots & C_{r}(2, N) \\
\vdots & \vdots & \ddots & \vdots \\
C_{r}(K, 1) & C_{r}(K, 2) & \cdots & C_{r}(K, N)
\end{array}\right),
$$

where $C_{r}(k, n)$ is the symbol transmitted by the $n$-th relay node on the $k$-th subcarrier. The SF is assumed to satisfy the power constraint $\left\|\mathbf{C}_{r}\right\|_{F}^{2} \leq K$.

The received signal at the destination node on the $k$-th subcarrier is given by

$$
y_{d}(k)=\sqrt{P_{r}} \sum_{n=1}^{N} H_{r_{n}, d}(k) C_{r}(k, n) I_{n}+\eta_{r_{n}, d}(k),
$$

where $P_{r}$ is the relay node power, $H_{r_{n}, d}(k)$ is the attenuation of the channel between the $n$-th relay node and the destination node on the $k$-th subcarrier, $\eta_{r_{n}, d}(k)$ is the destination additive white Gaussian noise on the $k$-th subcarrier, and $I_{n}$ is the state of the $n$-th relay. $I_{n}$ will equal 1 if the $n$-th relay has decoded correctly in phase 1 , otherwise, $I_{n}$ will equal 0 .

\section{B. Performance Analysis}

It is now necessary to develop sufficient code design criteria for the DSFC to achieve full diversity of order $N L$. Unlike the case of MIMO space-frequency coding, we will need a two-stage coding to achieve full diversity at the destination node. Therefore, the proposed DSFCs will have two stages of coding: the first stage is coding at the source node and the second stage is coding at the relay nodes. The transmitted source node code will be designed to guarantee a diversity of order $L$ at any relay node, and this will in turn cause the proposed DSFC to achieve full diversity of order $N L$ as will be shown later.

\footnotetext{
${ }^{2} \mathrm{FFT}$, which is used to transform the received data from the timedomain to the frequency-domain, can be represented by a unitary matrix multiplication. Unitary transformation of a Gaussian random vector, whose components are statistically independent and identically distributed, results in a Gaussian random vector with statistically independent and identically distributed components.

${ }^{3} \mathbf{C}_{r}$ will be SF code transmitted by the relay nodes if all of them have decoded correctly in phase 1 .
}

1) Source Node Coding: Due to the symmetry assumption, the pairwise error probability (PEP) is the same at any relay node. For two distinct transmitted source node symbols, $\mathbf{s}$ and $\tilde{\mathbf{s}}$, the PEP can be tightly upper bounded as [12], [8]

$$
P E P(\mathbf{s} \rightarrow \tilde{\mathbf{s}}) \leq\left(\begin{array}{c}
2 \nu-1 \\
\nu
\end{array}\right)\left(\prod_{i=1}^{\nu} \lambda_{i}\right)^{-1}\left(\frac{P_{s}}{N_{0}}\right)^{-\nu}
$$

and $\nu$ is the rank of the matrix $\mathbf{C} \circ \mathbf{R}$ where

$$
\begin{gathered}
\mathbf{C}=(\mathbf{s}-\tilde{\mathbf{s}})(\mathbf{s}-\tilde{\mathbf{s}})^{\mathcal{H}}, \\
\mathbf{R}=E\left\{\mathbf{H}_{s, r_{n}} \mathbf{H}_{s, r_{n}}^{\mathcal{H}}\right\},
\end{gathered}
$$

and $\mathbf{H}_{s, r_{n}}=\left[H_{s, r_{n}}(1), \cdots, H_{s, r_{n}}(K)\right]^{T}$. Here $\lambda_{i}$ 's are the non-zero eigenvalues of the matrix $\mathbf{C} \circ \mathbf{R}$, where $\circ$ denotes the Hadamard product ${ }^{4}$.

The correlation matrix, $\mathbf{R}$, of the channel impulse response can be found as

$$
\begin{aligned}
\mathbf{R} & =E\left\{\mathbf{H}_{s, r_{n}} \mathbf{H}_{s, r_{n}}^{\mathcal{H}}\right\} \\
& =\mathbf{W} E\left\{\alpha_{s, r_{n}} \alpha_{s, r_{n}}^{\mathcal{H}}\right\} \mathbf{W}^{\mathcal{H}} \\
& =\mathbf{W} \operatorname{diag}\left\{\sigma^{2}(1), \sigma^{2}(2), \cdots, \sigma^{2}(L)\right\} \mathbf{W}^{\mathcal{H}},
\end{aligned}
$$

where

$$
\begin{gathered}
\alpha_{s, r_{n}}=\left[\alpha_{s, r_{n}}(1), \alpha_{s, r_{n}}(2), \cdots, \alpha_{s, r_{n}}(L)\right]^{T} \\
\mathbf{W}=\left(\begin{array}{cccc}
1 & 1 & \cdots & 1 \\
w^{\tau_{1}} & w^{\tau_{2}} & \cdots & w^{\tau_{L}} \\
\vdots & \vdots & \ddots & \vdots \\
w^{(K-1) \tau_{1}} & w^{(K-1) \tau_{2}} & \cdots & w^{(K-1) \tau_{L}}
\end{array}\right),
\end{gathered}
$$

and $w=e^{-j 2 \pi \Delta f}$.

The coding at the source node is implemented to guarantee a diversity of order $L$, which is the maximum achievable diversity order at any relay node. We propose to partition the transmitted $K \times 1$ source node code into subblocks of length $L$ and we will design the subblocks to guarantee a diversity of order $L$ at any relay node as will be seen later. Let $M=\lfloor K / L\rfloor$ denote the number of subblocks in the source node transmitted OFDM block. The transmitted $K \times 1$ source node code is given as

$\mathbf{s}=[s(1), s(2), \cdots, s(K)]^{T}=\left[\mathbf{F}_{1}^{T}, \mathbf{F}_{2}^{T}, \cdots, \mathbf{F}_{M}^{T}, \mathbf{0}_{K-M L}^{T}\right]^{T}$,

where $\mathbf{F}_{i}=\left[F_{i}(1), \cdots, F_{i}(L)\right]^{T}$ is the $i$-th subblock of dimension $L \times 1$. Zeros are padded if $K$ is not an integer multiple of $L$. For any two distinct source codewords, $\mathbf{s}$ and $\tilde{\mathbf{s}}=\left[\tilde{\mathbf{F}}_{1}^{T}, \tilde{\mathbf{F}}_{2}^{T}, \cdots, \tilde{\mathbf{F}}_{M}^{T}, \mathbf{0}_{K-M L}^{T}\right]^{T}$, at least one index $p_{0}$ exists for which $\mathbf{F}_{p_{0}}$ is not equal to $\tilde{\mathbf{F}}_{p_{0}}$.

Based on the proposed structure of the transmitted code from the source node, sufficient conditions for the code to achieve a diversity of order $L$ at the relay nodes are derived. We assume for $\mathbf{s}$ and $\tilde{\mathbf{s}}$ that $\mathbf{F}_{p}=\tilde{\mathbf{F}}_{p}$ for all $p \neq p_{0}$, which corresponds to the worst-case PEP. This does not decrease the rank of the matrix $\mathbf{C} \circ \mathbf{R}$ [8]. Define the $L \times L$ matrix $\mathbf{Q}=\left\{q_{i, j}\right\}$ as $q_{i, j}=\sum_{l=1}^{L} \sigma^{2}(l) w^{(i-j) \tau(l)}, \quad 1 \leq i, j \leq L$. Note that the non-zero eigenvalues of the matrix $\mathbf{C} \circ \mathbf{R}$ are the

${ }^{4}$ If $\mathbf{A}=\left\{a_{i, j}\right\}$ and $\mathbf{B}=\left\{b_{i, j}\right\}$ are two $m \times n$ matrices, the Hadamard product is defined as $\mathbf{D}=\mathbf{A} \circ \mathbf{B}=\left\{d_{i, j}\right\}$, where $d_{i, j}=a_{i, j} b_{i, j}$. 


$$
\begin{aligned}
\left(\mathbf{F}_{p_{0}}-\tilde{\mathbf{F}}_{p_{0}}\right)\left(\mathbf{F}_{p_{0}}-\tilde{\mathbf{F}}_{p_{0}}\right)^{\mathcal{H}} \circ \mathbf{Q} & =\left[\operatorname{diag}\left(\mathbf{F}_{p_{0}}-\tilde{\mathbf{F}}_{p_{0}}\right) \mathbf{1}_{L \times L} \operatorname{diag}\left(\mathbf{F}_{p_{0}}-\tilde{\mathbf{F}}_{p_{0}}\right)^{\mathcal{H}}\right] \circ \mathbf{Q} \\
& =\operatorname{diag}\left(\mathbf{F}_{p_{0}}-\tilde{\mathbf{F}}_{p_{0}}\right) \mathbf{Q} \operatorname{diag}\left(\mathbf{F}_{p_{0}}-\tilde{\mathbf{F}}_{p_{0}}\right)^{\mathcal{H}}
\end{aligned}
$$

same as those of the matrix $\left(\mathbf{F}_{p_{0}}-\tilde{\mathbf{F}}_{p_{0}}\right)\left(\mathbf{F}_{p_{0}}-\tilde{\mathbf{F}}_{p_{0}}\right)^{\mathcal{H}} \circ \mathbf{Q}$. Hence, we have (9), where $\mathbf{1}_{L \times L}$ is the $L \times L$ matrix whose all elements are ones. The last equality follows from a property of the Hadamard product ([13], p.304).

If all of the eigenvalues of the matrix $\left(\mathbf{F}_{p_{0}}-\tilde{\mathbf{F}}_{p_{0}}\right)\left(\mathbf{F}_{p_{0}}-\tilde{\mathbf{F}}_{p_{0}}\right)^{\mathcal{H}} \circ \mathbf{Q}$ are non-zero, then their product can be calculated as shown in (10). The matrix $\mathbf{Q}$ is non-singular. Hence, if the product $\prod_{l=1}^{L}\left|F_{p_{0}}(l)-\tilde{F}_{p_{0}}(l)\right|^{2}$ is non-zero over all possible pairs of distinct transmitted source codewords, $\mathbf{s}$ and $\tilde{\mathbf{s}}$, then a diversity of order $L$ will be achieved at each relay node.

In phase 2, relays that have decoded correctly in phase 1 will forward the source node information. The received signal at the destination node on the $k$-th subcarrier is as given in (5). The state of the $n$-th relay node $I_{n}$ is a Bernoulli random variable with a probability mass function (pmf) given by

$$
I_{n}= \begin{cases}0 & \text { with probability }=S E R \\ 1 & \text { with probability }=1-S E R,\end{cases}
$$

where $S E R$ is the symbol error rate at the $n$-th relay node. Note that $S E R$ is the same for any relay node due to the symmetry assumption. If the transmitted code from the source node is designed such that the product $\prod_{l=1}^{L}\left|F_{p_{0}}(l)-\tilde{F}_{p_{0}}(l)\right|^{2}$ is non-zero, for at least one index $p_{0}$, over all the possible pairs of distinct transmitted source codewords, $\mathbf{s}$ and $\tilde{\mathbf{s}}$, then the $S E R$ at the $n$-th relay node can be upper bounded as

$$
\begin{aligned}
S E R & =\sum_{\mathbf{s} \in \mathcal{S}} \operatorname{Pr}\{\mathbf{s}\} \operatorname{Pr}\{\text { error given that } \mathbf{s} \text { was transmitted }\} \\
& \leq \sum_{\mathbf{s} \in \mathcal{S}} \operatorname{Pr}\{\mathbf{s}\} \sum_{\tilde{\mathbf{s}} \in \mathcal{S}, \tilde{\mathbf{s}} \neq \mathbf{s}} \operatorname{PEP}(\mathbf{s} \rightarrow \tilde{\mathbf{s}}) \\
& \leq c \times S N R^{-L},
\end{aligned}
$$

where $\mathcal{S}$ is the set of all possible transmitted source codewords and $c$ is a constant that does not depend on the $S N R$. The first inequality follows from the union upper bound and the second inequality follows from (6), where $S N R$ is defined as $S N R=P_{s} / N_{0}$.

2) Relay Nodes Coding: Next, the design of the SF code at the relay nodes to achieve a diversity of order $N L$ is considered. We propose to design SF codes constructed from the concatenation of block diagonal matrices, which is similar to the structure used in [8] to design full-rate, full-diversity space-frequency codes over MIMO channels. We will derive sufficient conditions for the proposed code structure to achieve full diversity at the destination node.

Let $P=\lfloor K / N L\rfloor$ denote the number of subblocks in the transmitted OFDM block from the relay nodes. The transmitted $K \times N$ SF codeword from the relay nodes, if all relays decoded correctly, is given by

$$
\mathbf{C}_{r}=\left[\mathbf{G}_{1}^{T}, \mathbf{G}_{2}^{T}, \cdots, \mathbf{G}_{P}^{T}, \mathbf{0}_{K-P L N}^{T}\right]^{T},
$$

where $\mathbf{G}_{i}$ is the $i$-th subblock of dimension $N L \times N$. Zeros are padded if $K$ is not an integer multiple of $N L$. Each $\mathbf{G}_{i}$ is a block diagonal matrix that has the structure

$$
\mathbf{G}_{i}=\left(\begin{array}{cccc}
\mathbf{X}_{1_{L \times 1}} & \mathbf{0}_{L \times 1} & \cdots & \mathbf{0}_{L \times 1} \\
\mathbf{0}_{L \times 1} & \mathbf{X}_{2_{L \times 1}} & \cdots & \mathbf{0}_{L \times 1} \\
\vdots & \vdots & \ddots & \vdots \\
\mathbf{0}_{L \times 1} & \mathbf{0}_{L \times 1} & \cdots & \mathbf{X}_{N_{L \times 1}}
\end{array}\right)
$$

and let $\mathbf{X}=\left[\mathbf{X}_{1}^{T}, \mathbf{X}_{2}^{T}, \cdots, \mathbf{X}_{N}^{T}\right]=[x(1), x(2), \cdots, x(N L)]$.

For two distinct transmitted source codewords, $\mathbf{s}$ and $\tilde{\mathbf{s}}$, and a given realization of the relays states $\mathbf{I}=\left[I_{1}, I_{2}, \cdots, I_{N}\right]^{T}$, the conditional PEP can be tightly upper bounded as

$$
P E P(\mathbf{s} \rightarrow \tilde{\mathbf{s}} / \mathbf{I}) \leq\left(\begin{array}{c}
2 \kappa-1 \\
\kappa
\end{array}\right)\left(\prod_{i=1}^{\kappa} \eta_{i}\right)^{-1}\left(\frac{P_{r}}{N_{0}}\right)^{-\kappa},
$$

and $\kappa$ is the rank of the matrix $\mathbf{C}(\mathbf{I}) \circ \mathbf{R}$ where

$$
\mathbf{C}(\mathbf{I})=\left(\mathbf{C}_{r}-\tilde{\mathbf{C}}_{r}\right) \operatorname{diag}(\mathbf{I})\left(\mathbf{C}-\tilde{\mathbf{C}}_{r}\right)^{\mathcal{H}} .
$$

For two source codewords, $\mathbf{s}$ and $\tilde{\mathbf{s}}$, at least one index $p_{0}$ exists for which $\mathbf{G}_{p_{0}} \neq \tilde{\mathbf{G}}_{p_{0}}$. We assume for $\mathbf{s}$ and $\tilde{\mathbf{s}}$ that $\mathbf{G}_{p}=\tilde{\mathbf{G}}_{p}$ for all $p \neq p_{0}$. Similar to the case of source node coding, this assumption does not decrease the rank of the matrix $\mathbf{C}(\mathbf{I}) \circ \mathbf{R}$ that corresponds to any realization $\mathbf{I}$ of the relays states.

Define the $N L \times N L$ matrix $\mathbf{S}=\left\{s_{i, j}\right\}$ as

$$
s_{i, j}=\sum_{l=1}^{L} \sigma^{2}(l) w^{(i-j) \tau(l)}, \quad 1 \leq i, j \leq N L .
$$

Note that the non-zero eigenvalues of the matrix $\mathbf{C}(\mathbf{I}) \circ \mathbf{R}$ are the same as the non-zero eigenvalues of the matrix $\left(\mathbf{G}_{p_{0}}(\mathbf{I})-\tilde{\mathbf{G}}_{p_{0}}(\mathbf{I})\right)\left(\mathbf{G}_{p_{0}}(\mathbf{I})-\tilde{\mathbf{G}}_{p_{0}}(\mathbf{I})\right)^{\mathcal{H}} \circ \mathbf{S}$ where $\mathbf{G}_{p_{0}}(\mathbf{I})$ is formed from $\mathbf{G}_{p_{0}}$ by setting the columns corresponding to the relays that have decoded erroneously to zeros. Hence, we have (16), where the second and the third equalities follow from the properties of the tensor and Hadamard products [13].

Let $n_{\mathbf{I}}=\sum_{n=1}^{N} I_{n}$ denote the number of relays that have decoded correctly corresponding to a realization $\mathbf{I}$ of the relays states. Using (16), the product of the non-zero eigenvalues of the matrix $\mathbf{C}(\mathbf{I}) \circ \mathbf{R}$ can be found as

$$
\prod_{i=1}^{\kappa} \eta_{i}=\left(\prod_{i=1, i \in \mathcal{I}}^{N L}|x(i)-\tilde{x}(i)|^{2}\right) \cdot\left(\operatorname{det}\left(\mathbf{S}_{0}\right)\right)^{n_{\mathbf{I}}}
$$

where $\mathcal{I}$ is the index set of symbols that are transmitted from the relays that have decoded correctly corresponding to the realization $\mathbf{I}$ and $\mathbf{S}_{0}=\left\{s_{i, j}\right\}, 1 \leq i, j \leq L$. The 


$$
\begin{aligned}
\operatorname{det}\left(\left(\mathbf{F}_{p_{0}}-\tilde{\mathbf{F}}_{p_{0}}\right)\left(\mathbf{F}_{p_{0}}-\tilde{\mathbf{F}}_{p_{0}}\right)^{\mathcal{H}} \circ \mathbf{Q}\right) & =\operatorname{det}\left(\operatorname{diag}\left(\mathbf{F}_{p_{0}}-\tilde{\mathbf{F}}_{p_{0}}\right)\right) \operatorname{det}(\mathbf{Q}) \operatorname{det}\left(\operatorname{diag}\left(\mathbf{F}_{p_{0}}-\tilde{\mathbf{F}}_{p_{0}}\right)^{\mathbf{H}}\right) \\
& =\prod_{l=1}^{L}\left|F_{p_{0}}(l)-\tilde{F}_{p_{0}}(l)\right|^{2}(\operatorname{det}(Q))
\end{aligned}
$$

$$
\begin{aligned}
& \left(\mathbf{G}_{p_{0}}(\mathbf{I})-\tilde{\mathbf{G}}_{p_{0}}(\mathbf{I})\right)\left(\mathbf{G}_{p_{0}}(\mathbf{I})-\tilde{\mathbf{G}}_{p_{0}}(\mathbf{I})\right)^{\mathcal{H}} \circ \mathbf{S} \\
& =\left(\operatorname{diag}(\mathbf{X}-\tilde{\mathbf{X}})\left(\operatorname{diag}(\mathbf{I}) \otimes \mathbf{1}_{L \times 1}\right)\left(\operatorname{diag}(\mathbf{I}) \otimes \mathbf{1}_{L \times 1}\right)^{\mathcal{H}} \operatorname{diag}(\mathbf{X}-\tilde{\mathbf{X}})^{\mathcal{H}}\right) \circ \mathbf{S} \\
& =\left(\operatorname{diag}(\mathbf{X}-\tilde{\mathbf{X}})\left(\operatorname{diag}(\mathbf{I}) \otimes \mathbf{1}_{L \times L}\right) \operatorname{diag}(\mathbf{X}-\tilde{\mathbf{X}})^{\mathcal{H}}\right) \circ \mathbf{S} \\
& =\operatorname{diag}(\mathbf{X}-\tilde{\mathbf{X}})\left[\left(\operatorname{diag}(\mathbf{I}) \otimes \mathbf{1}_{L \times L} \circ \mathbf{S}\right] \operatorname{diag}(\mathbf{X}-\tilde{\mathbf{X}})^{\mathcal{H}}\right.
\end{aligned}
$$

result in (17) is based on the assumption that the product $\prod_{i=1, i \in \mathcal{I}\}}^{N L}|x(i)-\tilde{x}(i)|^{2}$ is non-zero. The first product in (17) is over $n_{\mathbf{I}} L$ terms. The matrix $\mathbf{S}_{0}$ is always full rank of order $L$. Hence, designing the product $\prod_{i=1, i \in \mathcal{I}}^{N L}|x(i)-\tilde{x}(i)|^{2}$ to be non-zero will guarantee a rate of decay, at high SNR, of the conditional PEP as $S N R^{-n_{\mathrm{I}} L}$, where SNR is now defined as $S N R=P_{r} / N_{0}$. To guarantee that this rate of decay, $S N R^{-n_{\mathbf{I}} L}$, is always achieved irrespective of the state realization $\mathbf{I}$ of the relay nodes then the product $\prod_{i=1}^{N L}|x(i)-\tilde{x}(i)|^{2}$ should be non-zero. Hence, designing the product $\prod_{i=1}^{N L}|x(i)-\tilde{x}(i)|^{2}$ to be non-zero for any pair of distinct source codewords is a sufficient condition for the conditional PEP to decay as $S N R^{-n_{\mathbf{I}} L}$ for any realization $\mathbf{I}$, where $n_{\mathbf{I}}$ is the number of relays that have decoded correctly corresponding to $\mathbf{I}$.

Now, we calculate the PEP at the destination node for our proposed DSFC structure. Let $c_{r}$ denote the number of relays that have decoded correctly. Then $c_{r}$ follows a Binomial distribution as ${ }^{5}$

$$
\operatorname{Pr}\left\{c_{r}=k\right\}=\left(\begin{array}{c}
N \\
k
\end{array}\right)(1-S E R)^{k} S E R^{N-k},
$$

where $S E R$ is the symbol error rate at the relay nodes. The destination PEP is given by (19). Using the upper bound on the $S E R$ at the relay nodes given in (12) and the expression for the conditional PEP at the destination node in (15), and upper-bounding $(1-S E R)$ by 1 , it can be shown that

$$
P E P(\mathbf{s} \rightarrow \tilde{\mathbf{s}}) \leq \text { constant } \times S N R^{-N L} .
$$

Hence, our proposed structure for DSFCs with two-stage coding at the source node and the relay nodes achieves a diversity of order $N L$, which is the rate of decay of the PEP at high SNR.

\section{DSFC WITH THE AAF PROTOCOL}

In this section, the design and performance analysis for DSFCs with the AAF protocol are presented. A structure is proposed and sufficient conditions for the proposed structure to achieve full diversity are then derived for some special cases.

\footnotetext{
${ }^{5} c_{r}$ is a Binomial random variable as it is the sum of independent, identically distributed Bernoulli random variables.
}

\section{A. System Model}

In this section, we describe the system model for DSFC with the AAF protocol. The received signal model at the relay nodes and the channel gains are modeled as in Section II. The transmitted data from the source node is parsed into subblocks of size $N L \times 1$. Let $P=\lfloor K / N L\rfloor$ denote the number of subblocks in the transmitted OFDM block. The transmitted $K \times 1$ source codeword is given by

$$
\mathbf{s}=[s(1), s(2), \cdots, s(K)]^{T}=\left[\mathbf{B}_{1}^{T}, \mathbf{B}_{2}^{T}, \cdots, \mathbf{B}_{P}^{T}, \mathbf{0}_{K-P L N}^{T}\right]^{T},
$$

where $\mathbf{B}_{i}$ is the $i$-th subblock of dimension $N L \times 1$. Zeros are padded if $K$ is not an integer multiple of $N L$. For each subblock, $\mathbf{B}_{i}$, the $n$-th relay only forwards the data on $L$ subcarriers. For example, relay 1 will only forward $\left[\mathbf{B}_{i}(1), \cdots, \mathbf{B}_{i}(L)\right]$ for all $i$ 's and send zeros on the remaining set of subcarriers. In general, the $n$-th relay will only forward $\left[\mathbf{B}_{i}((n-1) L+1), \cdots, \mathbf{B}_{i}((n-1) L+L)\right]$ for all $i$ 's.

At the relay nodes, each node will normalize the received signal on the subcarriers that it will forward before retransmission and send zeros on the remaining set of subcarriers. If the $k$-th subcarrier is to be forwarded by the $n$-th relay, the relay will normalize the received signal on that subcarrier by the factor $\beta(k)=\sqrt{\frac{1}{P_{s}\left|H_{s, r_{n}}(k)\right|^{2}+N_{0}}}[3]$. The relay nodes will use OFDM modulation for transmission to the destination node. At the destination node, the received signal on the $k$ th subcarrier, assuming it was forwarded by the $n$-th relay, is given by (22), where $P_{r}$ is the relay node power, $H_{r_{n}, d}(k)$ is the attenuation of the channel between the $n$-th relay node and the destination node on the $k$-th subcarrier, and $\eta_{s, r_{n}}(k)$ is the destination noise on the $k$-th subcarrier. The $\eta_{r_{n}, d}(k)$ 's are modeled as zero mean, circularly symmetric complex Gaussian random variables with a variance of $N_{0} / 2$ per dimension.

\section{B. Performance Analysis}

In this section, the PEP of the DSFC with the AAF protocol is presented. Based on the PEP analysis, code design criteria are derived. The received signal at destination on the $k$-th subcarrier given by (22) can be rewritten as shown in (23), where $z_{r_{n}, d}(k)$ accounts for the noise propagating from the 


$$
\begin{gathered}
\operatorname{PEP}(\mathbf{s} \rightarrow \tilde{\mathbf{s}})=\sum_{\mathbf{I}} \operatorname{Pr}\{\mathbf{I}\} \operatorname{PEP}(\mathbf{s} \rightarrow \tilde{\mathbf{s}} / \mathbf{I}) \\
=\sum_{k=0}^{N} \operatorname{Pr}\left\{c_{r}=k\right\} \sum_{\left\{\mathbf{I}: n_{\mathbf{I}}=k\right\}} \operatorname{PEP}(\mathbf{s} \rightarrow \tilde{\mathbf{s}} / \mathbf{I}) \\
=\sum_{k=0}^{N}\left(\begin{array}{c}
N \\
k
\end{array}\right)(1-S E R)^{k} S E R^{N-k} \sum_{\left\{\mathbf{I}: n_{\mathbf{I}}=k\right\}} P E P(\mathbf{s} \rightarrow \tilde{\mathbf{s}} / \mathbf{I}) \\
y(k)=H_{r_{n}, d}(k) \sqrt{P_{r}}\left(\sqrt{\frac{1}{P_{s}\left|H_{s, r_{n}}(k)\right|^{2}+N_{0}}}\left(\sqrt{P_{s}} H_{s, r_{n}}(k) s(k)+\eta_{s, r_{n}}(k)\right)\right)+\eta_{r_{n}, d}(k), \\
y(k)=H_{r_{n}, d}(k) \sqrt{P_{r}}\left(\sqrt{\frac{1}{P_{s}\left|H_{s, r_{n}}(k)\right|^{2}+N_{0}}} \sqrt{P_{s}} H_{s, r_{n}}(k) s(k)\right)+z_{r_{n}, d}(k),
\end{gathered}
$$

relay node as well as the destination noise. $z_{r_{n}, d}(k)$ follows a circularly symmetric complex Gaussian random variable with a variance $\delta_{z}^{2}(k)$ of $\left(\frac{P_{r}\left|H_{r_{n}, d}(k)\right|^{2}}{P_{s}\left|H_{s, r_{n}}(k)\right|^{2}+N_{0}}+1\right) N_{0}$. The probability density function of $z_{r_{n}, d}(k)$ given the channel state information (CSI) is given by

$$
p\left(z_{r_{n}, d}(k) / \mathrm{CSI}\right)=\frac{1}{\pi \delta_{z}^{2}(k)} \exp \left(-\frac{1}{\delta_{z}^{2}(k)}\left|z_{r_{n}, d}(k)\right|^{2}\right) .
$$

The receiver applies a Maximum Likelihood (ML) detector to the received signal, which is given as

$\hat{\mathbf{s}}=\arg \min _{\mathbf{s}} \sum_{k=1}^{K} \frac{1}{\delta_{z}^{2}(k)}\left|\mathbf{y}(k)-\frac{\sqrt{P_{s} P_{r}} H_{s, r_{n}}(k) H_{r_{n}, d}(k)}{\sqrt{P_{s}\left|H_{s, r_{n}}(k)\right|^{2}+N_{0}}} \mathbf{s}(k)\right|^{2}$,

where the $n$ index (which is the index of the relay node) is adjusted according to the $k$ index (which is the index of the subcarrier).

Now, sufficient conditions for the proposed code structure to achieve full diversity are derived. The pdf of a received vector $\mathbf{y}=[y(1), y(2), \cdots, y(K)]^{T}$ given that the codeword $\mathbf{s}$ was transmitted is given by (26). The PEP of mistaking $\mathbf{s}$ by $\tilde{\mathbf{s}}$ can be upper bounded as [14]

$$
P E P(\mathbf{s} \rightarrow \tilde{\mathbf{s}}) \leq E\{\exp (\lambda[\ln p(\mathbf{y} / \tilde{\mathbf{s}})-\ln p(\mathbf{y} / \mathbf{s})])\},
$$

and the relation applies for any $\lambda$, which can selected to get the tightest bound. Any two distinct codewords $\mathbf{s}$ and $\tilde{\mathbf{s}}=$ $\left[\tilde{\mathbf{B}}_{1}, \tilde{\mathbf{B}}_{2}, \cdots, \tilde{\mathbf{B}}_{p}\right]^{T}$ will have at least one index $p_{0}$ such that $\tilde{\mathbf{B}}_{p_{0}} \neq \mathbf{B}_{p_{0}}$. We will assume that $\mathbf{s}$ and $\tilde{\mathbf{s}}$ will have only one index $p_{0}$ such that $\tilde{\mathbf{B}}_{p_{0}} \neq \mathbf{B}_{p_{0}}$, which corresponds to the worst case PEP.

Averaging the PEP expression in (27) over the noise distribution given in (24) we get (28), where $J=\left(p_{0}-\right.$ 1) $N L$. Take $\lambda=1 / 2$ to minimize the upper bound in (28), hence, we get (29). At high SNR, the term $\frac{P_{s}\left|H_{s, r_{n}}(k)\right|^{2} P_{r}\left|H_{r_{n}, d}(k)\right|^{2}}{\left(P_{s}\left|H_{s, r_{n}}(k)\right|^{2}+P_{r}\left|H_{r_{n}, d}(k)\right|^{2}+N_{0}\right) N_{0}}$ can be approximated by

$\frac{P_{s}\left|H_{s, r_{n}}(k)\right|^{2} P_{r}\left|H_{r_{n}, d}(k)\right|^{2}}{\left(P_{s}\left|H_{s, r_{n}}(k)\right|^{2}+P_{r}\left|H_{r_{n}, d}(k)\right|^{2}\right) N_{0}}$ [15], which is the scaled harmonic mean of the source-relay and relay-destination SNRs on the $k$-th subcarrier ${ }^{6}$. The scaled harmonic mean of two nonnegative numbers, $a_{1}$ and $a_{2}$, can be upper and lower bounded as

$$
\frac{1}{2} \min \left(a_{1}, a_{2}\right) \leq \frac{a_{1} a_{2}}{a_{1}+a_{2}} \leq \min \left(a_{1}, a_{2}\right) .
$$

Using the lower bound in (30) the PEP in (29) can be further upper bounded as shown in (31). If $P_{r}=P_{s}$ and $S N R$ is defined as $P_{s} / N_{0}$, then the PEP is now upper bounded as shown in (32).

1) PEP Analysis for $L=1$ : The case of $L$ equal to 1 corresponds to a flat, frequency nonselective fading channel. The PEP in (32) is now given by (33) It can be shown that the random variables $S N R\left|H_{s, r_{n}}(k)\right|^{2}$ and $S N R\left|H_{r_{n}, d}(k)\right|^{2}$ follow an exponential distribution with rate $1 / S N R$ for all $k$. The minimum of two exponential random variables is an exponential random variable with rate that is the sum of the two random variables rates. Hence, $\min \left(S N R\left|H_{s, r_{n}}(k)\right|^{2}, S N R\left|H_{r_{n}, d}(k)\right|^{2}\right)$ follows an exponential distribution with rate $2 / S N R$.

The PEP upper bound is now given by (34). At high SNR, we neglect the 1 term in the denominator of (34). Hence, the PEP can now be upper bounded as shown in (35). The result in (35) is under the assumption that the product $\prod_{n=1}^{N}\left|\mathbf{B}_{p_{0}}((n-1) L+1)-\tilde{\mathbf{B}}_{p_{0}}((n-1) L+1)\right|^{2}$ is non-zero. Clearly, if that product is non-zero, then the system will achieve a diversity of order $N L$, where $L$ is equal to 1 in this case. From the expression in (35) the coding gain of the space-frequency code is maximized when the product $\min _{\mathbf{s} \neq \tilde{\mathbf{s}}} \prod_{n=1}^{N}\left|\mathbf{B}_{p_{0}}((n-1) L+1)-\tilde{\mathbf{B}}_{p_{0}}((n-1) L+1)\right|^{2}$ is maximized. This product is known as the minimum product distance [8].

2) PEP Analysis for $L=2$ : The PEP in (32) can now be given as shown in (36), where $L=2$. The analysis in this case is more involved since the random variables appearing in (36) are correlated. Signals transmitted from the same

\footnotetext{
${ }^{6}$ The scaling factor is $1 / 2$ since the harmonic mean of two number, $X_{1}$ and $X_{2}$, is defined as $\frac{2 X_{1} X_{2}}{X_{1}+X_{2}}$.
} 


$$
p(\mathbf{y} / \mathbf{s}, \mathbf{C S I})=\left(\prod_{k=1}^{K} \frac{1}{\pi \delta_{z}^{2}(k)}\right) \exp \left(\sum_{k=1}^{K}-\frac{1}{\delta_{z}^{2}(k)}\left|y(k)-\frac{\sqrt{P_{s} P_{r}} H_{s, r_{n}}(k) H_{r_{n}, d}(k)}{\sqrt{P_{s}\left|H_{s, r_{n}}(k)\right|^{2}+N_{0}}} \mathbf{s}(k)\right|^{2}\right) .
$$

$$
\begin{aligned}
P E P(\mathbf{s} \rightarrow \tilde{\mathbf{s}}) \leq & E\left\{\operatorname { e x p } \left(-\lambda(1-\lambda) \sum_{n=1}^{N} \sum_{l=1}^{L}\right.\right. \\
& \left(\frac{P_{s}\left|H_{s, r_{n}}(J+(n-1) L+l)\right|^{2} P_{r}\left|H_{r_{n}, d}(J+(n-1) L+l)\right|^{2}}{\left(P_{s}\left|H_{s, r_{n}}(J+(n-1) L+l)\right|^{2}+P_{r}\left|H_{r_{n}, d}(J+(n-1) L+l)\right|^{2}+N_{0}\right) N_{0}}\right) \\
& \left.\left.\times\left|\mathbf{B}_{p_{0}}((n-1) L+l)-\tilde{\mathbf{B}}_{p_{0}}((n-1) L+l)\right|^{2}\right)\right\},
\end{aligned}
$$

$$
\begin{aligned}
P E P(\mathbf{s} \rightarrow \tilde{\mathbf{s}}) \leq & E\left\{\operatorname { e x p } \left(-\frac{1}{4} \sum_{n=1}^{N} \sum_{l=1}^{L}\right.\right. \\
& \left(\frac{P_{s}\left|H_{s, r_{n}}(J+(n-1) L+l)\right|^{2} P_{r}\left|H_{r_{n}, d}(J+(n-1) L+l)\right|^{2}}{\left(P_{s}\left|H_{s, r_{n}}(J+(n-1) L+l)\right|^{2}+P_{r}\left|H_{r_{n}, d}(J+(n-1) L+l)\right|^{2}+N_{0}\right) N_{0}}\right) \\
& \left.\left.\times\left|\mathbf{B}_{p_{0}}((n-1) L+l)-\tilde{\mathbf{B}}_{p_{0}}((n-1) L+l)\right|^{2}\right)\right\},
\end{aligned}
$$

$$
\left.\left.\left.\frac{P_{r}}{N_{0}}\left|H_{r_{n}, d}\left(\left(p_{0}-1\right) N L+(n-1) L+l\right)\right|^{2}\right)\left|\mathbf{B}_{p_{0}}((n-1) L+l)-\tilde{\mathbf{B}}_{p_{0}}((n-1) L+l)\right|^{2}\right)\right\}
$$

$P E P(\mathbf{s} \rightarrow \tilde{\mathbf{s}}) \leq E\left\{\exp \left(-\frac{1}{8} \sum_{n=1}^{N} \sum_{l=1}^{L} \min \left(S N R\left|H_{s, r_{n}}\left(\left(p_{0}-1\right) N L+(n-1) L+l\right)\right|^{2}\right.\right.\right.$,

$$
\left.\left.\left.S N R\left|H_{r_{n}, d}\left(\left(p_{0}-1\right) N L+(n-1) L+l\right)\right|^{2}\right)\left|\mathbf{B}_{p_{0}}((n-1) L+l)-\tilde{\mathbf{B}}_{p_{0}}((n-1) L+l)\right|^{2}\right)\right\}
$$

$$
\begin{aligned}
P E P(\mathbf{s} \rightarrow \tilde{\mathbf{s}}) & \leq E\left\{\operatorname { e x p } \left(-\frac{1}{8} \sum_{n=1}^{N} \min \left(S N R\left|H_{s, r_{n}}\left(\left(p_{0}-1\right) N L+(n-1) L+1\right)\right|^{2},\right.\right.\right. \\
& \left.\left.\left.S N R\left|H_{r_{n}, d}\left(\left(p_{0}-1\right) N L+(n-1) L+1\right)\right|^{2}\right)\left|\mathbf{B}_{p_{0}}((n-1) L+1)-\tilde{\mathbf{B}}_{p_{0}}((n-1) L+1)\right|^{2}\right)\right\} .
\end{aligned}
$$

$$
P E P(\mathbf{s} \rightarrow \tilde{\mathbf{s}}) \leq \prod_{n=1}^{N} \frac{1}{1+\frac{1}{16} S N R\left|\mathbf{B}_{p_{0}}((n-1) L+1)-\tilde{\mathbf{B}}_{p_{0}}((n-1) L+1)\right|^{2}}
$$

$$
P E P(\mathbf{s} \rightarrow \tilde{\mathbf{s}}) \lesssim\left(\frac{1}{16} S N R\right)^{-N}\left(\prod_{n=1}^{N}\left|\mathbf{B}_{p_{0}}((n-1) L+1)-\tilde{\mathbf{B}}_{p_{0}}((n-1) L+1)\right|^{2}\right)^{-1}
$$




$$
\begin{aligned}
& P E P(\mathbf{s} \rightarrow \tilde{\mathbf{s}}) \leq E\left\{\operatorname { e x p } \left(-\frac{1}{8} \sum_{n=1}^{N} \sum_{l=1}^{2} \min \left(S N R\left|H_{s, r_{n}}\left(\left(p_{0}-1\right) N L+(n-1) L+l\right)\right|^{2},\right.\right.\right. \\
& \left.\left.\left.S N R\left|H_{r_{n}, d}\left(\left(p_{0}-1\right) N L+(n-1) L+l\right)\right|^{2}\right)\left|\mathbf{B}_{p_{0}}((n-1) L+l)-\tilde{\mathbf{B}}_{p_{0}}((n-1) L+l)\right|^{2}\right)\right\}
\end{aligned}
$$

relay node on different subcarriers will experience correlated channel attenuations. As a first step in deriving the code design criterion, we prove that the channel attenuations, $\left|H_{s, r_{n}}\left(k_{1}\right)\right|^{2}$ and $\left|H_{s, r_{n}}\left(k_{2}\right)\right|^{2}$ for any $k_{1} \neq k_{2}$, have a bivariate Gamma distribution as their joint pdf [16]. The same applies for $\left|H_{r_{n}, d}\left(k_{1}\right)\right|^{2}$ and $\left|H_{r_{n}, d}\left(k_{2}\right)\right|^{2}$ for any $k_{1} \neq k_{2}$. The proof of this result is given in the Appendix.

To evaluate the expectation in (36) we need the expression for the joint pdf of the two random variables $M_{1}=\min \left(S N R\left|H_{s, r_{n}}\left(k_{1}\right)\right|^{2}, S N R\left|H_{r_{n}, d}\left(k_{1}\right)\right|^{2}\right)$ and $M_{2}=\min \left(S N R\left|H_{s, r_{n}}\left(k_{2}\right)\right|^{2}, S N R\left|H_{r_{n}, d}\left(k_{2}\right)\right|^{2}\right)$ for some $k_{1} \neq k_{2}$. Although $M_{1}$ and $M_{2}$ can be easily seen to be marginally exponential random variables, they are not jointly Gamma distributed. Define the random variables $X_{1}=S N R\left|H_{s, r_{n}}\left(k_{1}\right)\right|^{2}, X_{2}=S N R\left|H_{s, r_{n}}\left(k_{2}\right)\right|^{2}, Y_{1}=$ $S N R\left|H_{r_{n}, d}\left(k_{1}\right)\right|^{2}$, and $Y_{2}=S N R\left|H_{r_{n}, d}\left(k_{2}\right)\right|^{2}$. All of these random variables are marginally exponential with rate $1 / S N R$. Under the assumptions of our channel model, the pairs $\left(X_{1}, X_{2}\right)$ and $\left(Y_{1}, Y_{2}\right)$ are independent. Hence, the joint pdf of $\left(X_{1}, X_{2}, Y_{1}, Y_{2}\right)$, using the result in the Appendix, is given by (37), where $I_{0}(\cdot)$ is the modified Bessel function of the first kind of order zero and $U(\cdot)$ is the Heaviside unit step function [17]. $\rho_{x_{1} x_{2}}$ is the correlation coefficient between $X_{1}$ and $X_{2}$ and similarly, $\rho_{y_{1} y_{2}}$ is the correlation coefficient between $Y_{1}$ and $Y_{2}$. The joint cumulative distribution function (cdf) of the pair $\left(M_{1}, M_{2}\right)$ can be computed as shown in (38), where we have used the symmetry assumption of the source-relay and relay-destination channels. The joint pdf of $\left(M_{1}, M_{2}\right)$ can now be given as shown in (39). To get the PEP upperbound in (36) we need to calculate the expectation as shown in (40). At high enough SNR $I_{0}\left(\frac{2 \sqrt{\rho_{x_{1} x_{2}}}}{S N R\left(1-\rho_{x_{1} x_{2}}\right)} \sqrt{x_{1} x_{2}}\right)$ can be approximated to be 1 [17]. Using this approximation, the PEP upper bound can be approximated at high SNR as

$$
\begin{aligned}
& \operatorname{PEP}(\mathbf{s} \rightarrow \tilde{\mathbf{s}}) \\
& \lesssim\left(\prod_{m=1}^{2 N}\left|\mathbf{B}_{p_{0}}(m)-\tilde{\mathbf{B}}_{p_{0}}(m)\right|^{2}\right)^{-1}\left(\frac{1}{16}(1-\rho) S N R\right)^{-2 N},
\end{aligned}
$$

where $\rho=\rho_{x_{1} x_{2}}=\rho_{y_{1} y_{2}}$. Again, full diversity is achieved when the product $\prod_{m=1}^{2 N}\left|\mathbf{B}_{p_{0}}(m)-\tilde{\mathbf{B}}_{p_{0}}(m)\right|^{2}$ is non-zero. The coding gain of the space-frequency code is maximized when the product $\min _{\mathbf{s} \neq \tilde{\mathbf{s}}} \prod_{m=1}^{2 N}\left|\mathbf{B}_{p_{0}}(m)-\tilde{\mathbf{B}}_{p_{0}}(m)\right|^{2}$ is maximized.

The analysis becomes highly involved for any $L \geq 3$. It is very difficult to get closed form expressions in this case due to the correlation among the summed terms in (32) for which there is no closed form pdf expressions, similar to (37), are known [18].

\section{Code Construction And Discussions}

A construction method for the proposed DSFCs is presented here. This construction is the one used to do the source node and relay nodes coding for DSFCs with the DAF protocol. It is also used for designing DSFCs with the AAF protocol.

A linear mapping is used to form the transmitted subblocks, $\mathbf{D}=\mathbf{V}_{T \times T} \mathbf{s}_{\mathbf{G}}$, where $\mathbf{s}_{\mathbf{G}}$ is a $T \times 1$ source symbols vector transmitted in the subblock $\mathbf{D}$. $\mathbf{s}_{\mathbf{G}}$ is carved from QAM or PSK constellations. The goal is to maximize the product $\min _{\mathbf{s} \neq \tilde{\mathbf{s}}} \prod_{m=1}^{T}|\mathbf{D}(m)-\tilde{\mathbf{D}}(m)|^{2}$, where s and $\tilde{\mathbf{s}}$ are two distinct source symbols vectors. It was proposed in [19] and [20] to use both Hadamard transforms and Vandermonde matrices to design the $\mathbf{V}_{T \times T}$ matrix. The transforms based on the Vandermonde matrices proved to give larger minimum product distance than the Hadamard based transforms. Two classes of optimum transforms were proposed in [19] as follows

1) If $T=2^{k}(k \geq 1)$, the optimum transform is given by $\mathbf{V}_{\text {opt }}=\frac{1}{\sqrt{T}} \operatorname{vander}\left(\theta_{1}, \theta_{2}, \ldots, \theta_{T}\right)$, where $\theta_{1}, \theta_{2}, \ldots, \theta_{T}$ are $\theta_{n}=e^{j \frac{4 n-3}{2 T} \pi}, \quad n=1,2, \ldots, T$.

2) If $T=3.2^{k}(k \geq 0)$, the optimum transform is given by $\mathbf{V}_{\text {opt }}=\frac{1}{\sqrt{T}} \operatorname{vander}\left(\theta_{1}, \theta_{2}, \ldots, \theta_{T}\right)$, where $\theta_{1}, \theta_{2}, \ldots, \theta_{T}$ are $\theta_{n}=e^{j \frac{6 n-1}{3 T} \pi}, \quad n=1,2, \ldots, T$.

It noteworthy that the proposed DSFCs for both the DAF and AAF protocols achieve a data rate of $K / 2$ symbols/OFDM block, where $K$ is the number of subcarriers. The $1 / 2$ factor loss is due to the two-phase nature of the DAF and AAF protocols.

\section{A. Remarks}

Here we summarize some remarks related to our proposed DSFCs.

- Remark 1: In our problem formulation, we have considered a two-hop system model that lacks a direct link from the source node to the destination node. If such a direct link between the source node and the destination node exists, then the destination node can use its received signal from the source node to help recovering the source symbols. Assuming that the channel from the source node to the destination node has $L$ paths, it can be shown that our proposed DSFCs, with the proposed coding at the source node and the relay nodes for both the DAF and AAF protocols, achieve a diversity of order $(N+1) L$.

- Remark 2: The proposed DSFCs with the DAF protocol can be easily modified to achieve full diversity for the asymmetric case where the number of paths per fading channel is not the same for all channels. Let $L_{s, r_{n}}$ denote the number of paths of the channel between the source 


$$
\begin{aligned}
& f_{X_{1}, X_{2}, Y_{1}, Y_{2}}\left(x_{1}, x_{2}, y_{1}, y_{2}\right) \\
& =f_{X_{1}, X_{2}}\left(x_{1}, x_{2}\right) f_{Y_{1}, Y_{2}}\left(y_{1}, y_{2}\right) \\
& =\frac{1}{S N R^{2}\left(1-\rho_{x_{1} x_{2}}\right)\left(1-\rho_{y_{1} y_{2}}\right)} \exp \left(-\frac{x_{1}+x_{2}}{S N R\left(1-\rho_{x_{1} x_{2}}\right)}\right) I_{0}\left(\frac{2 \sqrt{\rho_{x_{1} x_{2}}}}{S N R\left(1-\rho_{x_{1} x_{2}}\right)} \sqrt{x_{1} x_{2}}\right) \\
& \times \exp \left(-\frac{y_{1}+y_{2}}{S N R\left(1-\rho_{y_{1} y_{2}}\right)}\right) I_{0}\left(\frac{2 \sqrt{\rho_{y_{1} y_{2}}}}{S N R\left(1-\rho_{y_{1} y_{2}}\right)} \sqrt{y_{1} y_{2}}\right) U\left(x_{1}\right) U\left(x_{2}\right) U\left(y_{1}\right) U\left(y_{2}\right), \\
& F_{M_{1}, M_{2}}\left(m_{1}, m_{2}\right) \triangleq \operatorname{Pr}\left[M_{1} \leq m_{1}, M_{2} \leq m_{2}\right] \\
& =\operatorname{Pr}\left[\min \left(X_{1}, Y_{1}\right) \leq m_{1}, \min \left(X_{2}, Y_{2}\right) \leq m_{2}\right] \\
& =2 \int_{y_{1}=0}^{m_{1}} \int_{x_{1}=y_{1}}^{\infty} \int_{y_{2}=0}^{m_{2}} \int_{x_{2}=y_{2}}^{\infty} f_{X_{1}, X_{2}}\left(x_{1}, x_{2}\right) f_{Y_{1}, Y_{2}}\left(y_{1}, y_{2}\right) d y_{1} d x_{1} d y_{2} d x_{2} \\
& +2 \int_{y_{1}=0}^{m_{1}} \int_{x_{1}=y_{1}}^{\infty} \int_{x_{2}=0}^{m_{2}} \int_{y_{2}=x_{2}}^{\infty} f_{X_{1}, X_{2}}\left(x_{1}, x_{2}\right) f_{Y_{1}, Y_{2}}\left(y_{1}, y_{2}\right) d y_{1} d x_{1} d x_{2} d y_{2} \\
& f_{M_{1}, M_{2}}\left(m_{1}, m_{2}\right)=\frac{\partial^{2}}{\partial m_{1} \partial m_{2}} F_{M_{1}, M_{2}}\left(m_{1}, m_{2}\right) \\
& =2 f_{Y_{1}, Y_{2}}\left(m_{1}, m_{2}\right) \int_{x_{1}=m_{1}}^{\infty} \int_{x_{2}=m_{2}}^{\infty} f_{X_{1}, X_{2}}\left(x_{1}, x_{2}\right) d x_{1} d x_{2} \\
& +2 \int_{x_{1}=m_{1}}^{\infty} \int_{y_{2}=m_{2}}^{\infty} f_{X_{1}, X_{2}}\left(x_{1}, m_{2}\right) f_{Y_{1}, Y_{2}}\left(m_{1}, y_{2}\right) d x_{1} d x_{2} . \\
& \begin{array}{l}
E\left\{\exp \left(-\frac{1}{8}\left(M_{1}\left|\mathbf{B}\left(k_{1}\right)-\tilde{\mathbf{B}}\left(k_{1}\right)\right|^{2}+M_{2}\left|\mathbf{B}\left(k_{2}\right)-\tilde{\mathbf{B}}\left(k_{2}\right)\right|^{2}\right)\right)\right\} \\
\quad=\int_{m_{1}=0}^{\infty} \int_{m_{2}=0}^{\infty} \exp \left(-\frac{1}{8}\left(m_{1}\left|\mathbf{B}\left(k_{1}\right)-\tilde{\mathbf{B}}\left(k_{1}\right)\right|^{2}+m_{2}\left|\mathbf{B}\left(k_{2}\right)-\tilde{\mathbf{B}}\left(k_{2}\right)\right|^{2}\right)\right) f_{M_{1}, M_{2}}\left(m_{1}, m_{2}\right) d m_{1} d m_{2}
\end{array}
\end{aligned}
$$

node and $n$-th relay and $L_{r_{n}, d}$ denote the number of paths of the channel between the $n$-th relay node and the destination node. The proposed DSFC can be easily modified to achieve a diversity $d$ of order

$$
d=\sum_{n=1}^{N} \min \left(L_{s, r_{n}}, L_{r_{n}, d}\right)
$$

which can be easily shown to be the maximal achievable diversity order. This maximal diversity order can be achieved, for example, by designing the codes at the source node and relay nodes using $L=$ $\max _{n} \min \left(L_{s, r_{n}}, L_{r_{n}, d}\right)$.

- Remark 3: The proposed construction for the design of DSFCs can be easily generalized to the case of multiantenna nodes, where any node may have more than one antenna. Each antenna can be treated as a separate relay node and the analysis presented before directly applies.

- Remark 4: As mentioned before, the presence of the cyclic prefix in the OFDM transmission provides a mean for combating the relays synchronization mismatches. Hence, our proposed DSFCs, which are based on OFDM transmission, are robust against synchronization mismatches within the duration of the cyclic prefix.

\section{Simulation Results}

In this section, some simulation results for the proposed DSFCs are presented. We will compare the performance of DSFCs with the DAF protocol to DSFCs with the AAF protocol. In all simulations, the source is assumed to have two relay nodes helping to forward its information. We use the two-hop channel model presented in the previous sections.

Fig. 2 shows the case of a simple two-ray, $L=2$, channel model with a delay of $\tau=5 \mu \mathrm{sec}$ between the two rays. The two rays have equal powers, i.e., $\sigma^{2}(1)=\sigma^{2}(2)$. The number of subcarriers is $K=128$ with a system bandwidth of $1 \mathrm{MHz}$. We use BPSK modulation and Vandermonde based linear transformations. Fig. 2 shows the SER of the proposed DSFCs versus the SNR defined as $S N R=\frac{P_{s}+P_{r}}{N_{0}}$, and we use $P_{s}=P_{r}$, i.e., equal power allocation between the source and relay nodes. We simulated three cases: all channel variances are ones, relays close to source, and relays close to destination. For the case of relays close to source, the variance of any source-relay channel is taken to be 10 and the variance of any relay-destination channel is taken to be 1 . For the case of relays close to destination, the variance of any sourcerelay channel is taken to be 1 and the variance of any relaydestination channel is taken to be 10. From Fig. 2, it is clear that DSFCs with the DAF protocol have a better performance than DSFCs with the AAF protocol. The reason is that DSFCs 


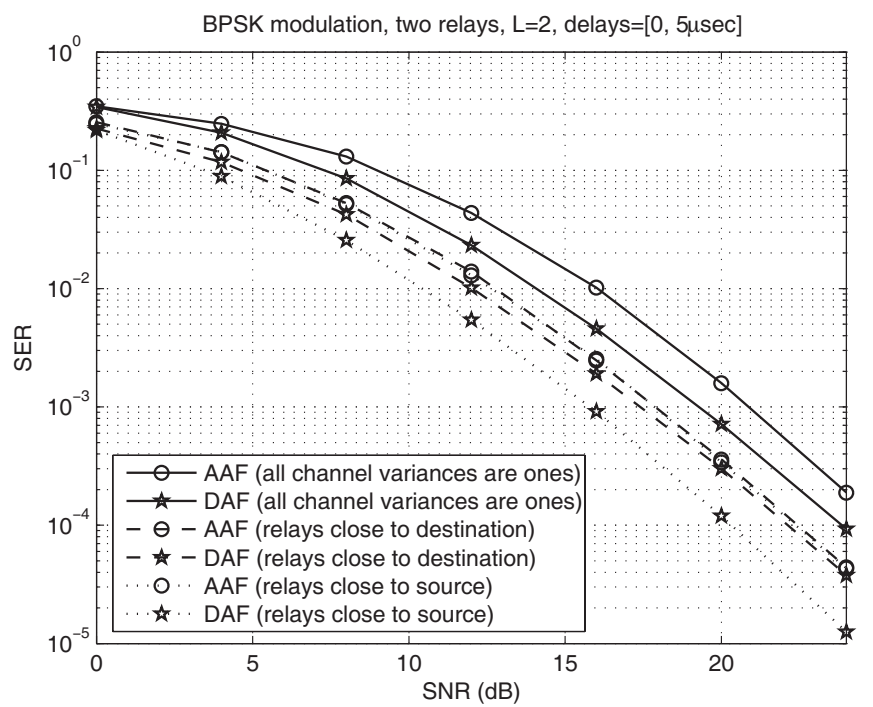

Fig. 2. SER of DSFCs for BPSK modulation, $L=2$, and delay $=[0,5 \mu \mathrm{sec}]$ versus SNR.

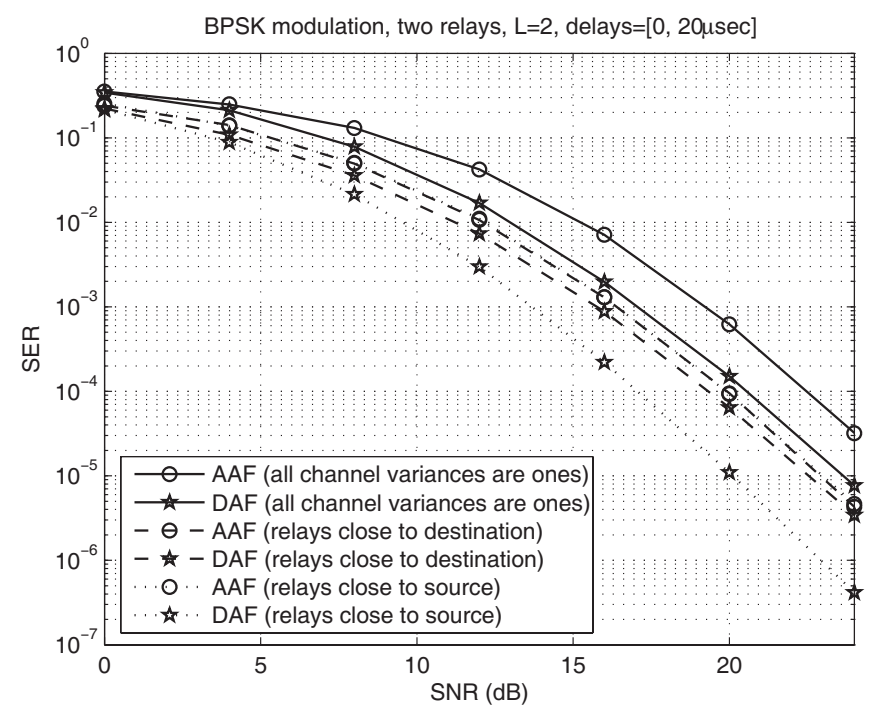

Fig. 3. SER of DSFCs for BPSK modulation, $L=2$, and delay $=[0,20 \mu \mathrm{sec}]$ versus SNR.

with DAF protocol deliver a less noisy code to the destination node as compared to DSFCs with AAF protocol, where noise propagation results from the transmissions of the relay nodes. Decoding at the relay nodes, in the DAF protocol, has the effect of removing the noise before retransmission to the destination node. As can be seen from Fig. 2, a gain of about $3 \mathrm{~dB}$ is achieved, for the case of relays close to the source, by employing DSFCs with the DAF protocol as compared to DSFCs with the AAF protocol.

Fig. 3 shows the case of a simple two-ray, $L=2$, with a delay of $\tau=20 \mu \mathrm{sec}$ between the two rays. The simulation setup is the same as that used in Fig. 2. From Fig. 3, it is clear that DSFCs with the DAF protocol have a better performance than DSFCs with the AAF protocol.

Fig. 4 shows the case of $L=4$ with a path delay vector given by $[0,5 \mu \mathrm{sec}, 10 \mu \mathrm{sec}, 15 \mu \mathrm{sec}]$. The rays are assumed to be of equal powers, i.e., $\sigma^{2}(l)=\sigma^{2}, l=1, \cdots, 4$. The

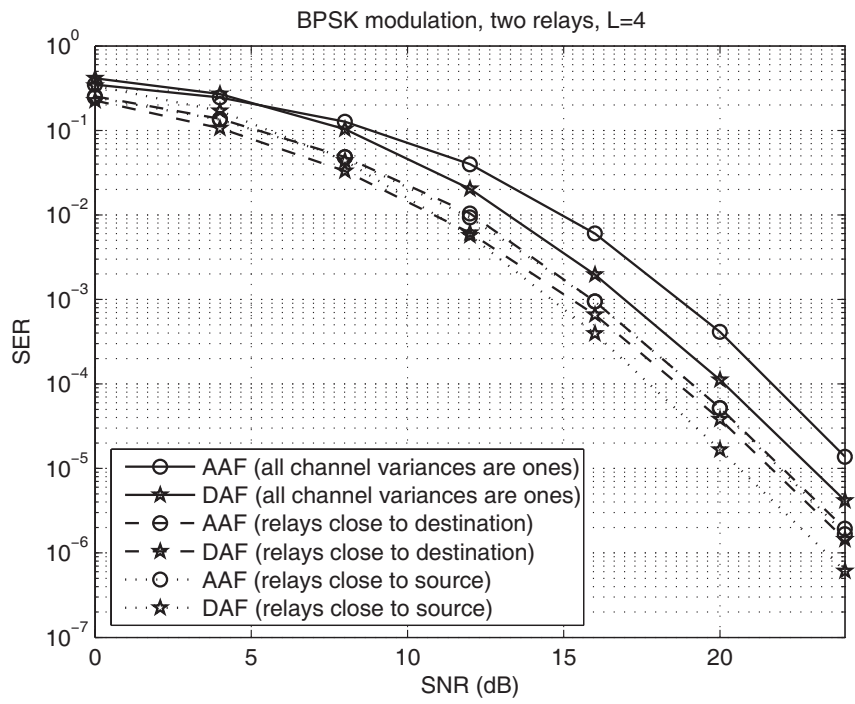

Fig. 4. SER of DSFCs for BPSK modulation, $L=4$, and delay $=[0,5 \mu \mathrm{sec}$, $10 \mu \mathrm{sec}, 15 \mu \mathrm{sec}]$ versus SNR.

number of subcarriers is $K=128$ with a system bandwidth of $1 \mathrm{MHz}$. We use BPSK modulation and Vandermonde based linear transformations. Fig. 4 shows the SER of the proposed DSFCs versus the SNR defined as $S N R=\frac{P_{s}+P_{r}}{N_{0}}$ and again we use $P_{s}=P_{r}$. We have simulated the same three cases as in Fig. 2. Fig. 4 shows that DSFCs with the DAF protocol have a better performance than DSFCs with the AAF protocol. We can observe a gain of about $2 \mathrm{~dB}$ for the case of relays close to source.

\section{CONCLUSIONS}

In this paper, the design of distributed space-frequency codes (DSFCs) was considered for the wireless multipath relay channels. The use of DSFCs can greatly improve system performance by achieving higher diversity orders by exploiting the multipath diversity of the channel as well as the cooperative diversity. In this paper, we have considered the design of DSFCs with the DAF and AAF cooperation protocols. For the case of DSFCs with the DAF protocol, we have proposed a two-stage coding scheme: source node coding and relay nodes coding. We have derived sufficient conditions for the proposed code structure to achieve full diversity of order $N L$ where $N$ is the number of relay nodes and $L$ is the number of multipaths per channel. For the case of DSFCs with the AAF protocol, we have derived sufficient conditions for the proposed code structure to achieve full diversity of order $N L$ for the special cases of $L=1$ and $L=2$.

Simulation results indicate that the performance of DSFCs with the DAF protocol is better that DSFCs with the AAF protocol because the decoding at the relay nodes can provide a mean for removing the noise from the relay transmitted signal. Hence, the relay provides the destination with a cleaner version of the code in the DAF protocol while the system suffers from noise propagation if the AAF protocol is used.

The proposed DSFCs are robust against the synchronization errors caused by the relays timing mismatches and propagation delays due to the presence of the cyclic prefix in the OFDM 


$$
H_{s, r_{n}}\left(k_{1}\right)=\alpha_{s, r_{n}}(1)+\alpha_{s, r_{n}}(2) e^{-j 2 \pi\left(k_{1}-1\right) \Delta f \tau_{2}}=\Re\left(H_{s, r_{n}}\left(k_{1}\right)\right)+j \Im\left(H_{s, r_{n}}\left(k_{1}\right)\right)
$$

$$
\begin{aligned}
& \Re\left(H_{s, r_{n}}\left(k_{1}\right)\right)=\Re\left(\alpha_{s, r_{n}}(1)\right)+\Re\left(\alpha_{s, r_{n}}(2)\right) \cos \left(2 \pi\left(k_{1}-1\right) \Delta f \tau_{2}\right)+\Im\left(\alpha_{s, r_{n}}(2)\right) \sin \left(2 \pi\left(k_{1}-1\right) \Delta f \tau_{2}\right) \\
& \Im\left(H_{s, r_{n}}\left(k_{1}\right)\right)=\Im\left(\alpha_{s, r_{n}}(1)\right)+\Im\left(\alpha_{s, r_{n}}(2)\right) \cos \left(2 \pi\left(k_{1}-1\right) \Delta f \tau_{2}\right)-\Re\left(\alpha_{s, r_{n}}(2)\right) \sin \left(2 \pi\left(k_{1}-1\right) \Delta f \tau_{2}\right)
\end{aligned}
$$

transmission. This property of the proposed DSFCs greatly simplifies the system design since it is very difficult to synchronize randomly located relay nodes.

\section{APPENDIX}

Consider the two random variables $H_{s, r_{n}}\left(k_{1}\right)$ and $H_{s, r_{n}}\left(k_{2}\right)$, we will assume without loss of generality that $\tau_{1}=$ 0 , i.e., the delay of the first path is zero. $H_{s, r_{n}}\left(k_{1}\right)$ is given by (42), where $\Re(x)$, and $\Im(x)$ are the real, and imaginary parts of $x$, respectively. From (42) we have (43). Based on the channel model presented in Section II-A both $\Re\left(H_{s, r_{n}}\left(k_{1}\right)\right)$ and $\Im\left(H_{s, r_{n}}\left(k_{1}\right)\right)$ are zero-mean Gaussian random variables with variance $1 / 2$. The correlation coefficient, $\rho_{r i}$, between $\Re\left(H_{s, r_{n}}\left(k_{1}\right)\right)$ and $\Im\left(H_{s, r_{n}}\left(k_{1}\right)\right)$ can be calculated as

$$
\rho_{r i}=E\left\{\Re\left(H_{s, r_{n}}\left(k_{1}\right)\right) \Im\left(H_{s, r_{n}}\left(k_{1}\right)\right)\right\}=0 .
$$

Hence, $H_{s, r_{n}}\left(k_{1}\right)$ is a circularly symmetric complex Gaussian random variable with variance $1 / 2$ per dimension and the same applies for $H_{s, r_{n}}\left(k_{2}\right)$. To get the joint probability distribution of $\left|H_{s, r_{n}}\left(k_{1}\right)\right|^{2}$ and $\left|H_{s, r_{n}}\left(k_{2}\right)\right|^{2}$, we can use the standard techniques of transformation of random variables. Using transformation of random variables and the fact that both $H_{s, r_{n}}\left(k_{1}\right)$ and $H_{s, r_{n}}\left(k_{2}\right)$ are circularly symmetric complex Gaussian random variables, it can be shown that $X_{1}=\left|H_{s, r_{n}}\left(k_{1}\right)\right|^{2}$ and $X_{2}=\left|H_{s, r_{n}}\left(k_{2}\right)\right|^{2}$ are jointly distributed according to a bivariate Gamma distribution with pdf [18], [16]

$$
\begin{gathered}
f_{X_{1}, X_{2}}\left(x_{1}, x_{2}\right)=\frac{1}{1-\rho_{x_{1} x_{2}}} \exp \left(-\frac{x_{1}+x_{2}}{1-\rho_{x_{1} x_{2}}}\right) \\
I_{0}\left(\frac{2 \sqrt{\rho_{x_{1} x_{2}}}}{1-\rho_{x_{1} x_{2}}} \sqrt{x_{1} x_{2}}\right) U\left(x_{1}\right) U\left(x_{2}\right),
\end{gathered}
$$

where $I_{0}(\cdot)$ is the modified Bessel function of the first kind of order zero and $U(\cdot)$ is the Heaviside unit step function [17]. $\rho_{x_{1} x_{2}}$ is the correlation between $\left|H_{s, r_{n}}\left(k_{1}\right)\right|^{2}$ and $\left|H_{s, r_{n}}\left(k_{2}\right)\right|^{2}$ and it can calculated as

$$
\rho_{x_{1}, x_{2}}=\frac{\operatorname{Cov}\left(X_{1}, X_{2}\right)}{\sqrt{\operatorname{Var}\left(X_{1}\right) \operatorname{Var}\left(X_{2}\right)}} .
$$

Following tedious computations, it can be shown that

$$
\rho_{x_{1}, x_{2}}=\frac{1}{2}+2 \sigma^{2}(1) \sigma^{2}(2) \cos \left(2 \pi\left(k_{2}-k_{1}\right) \Delta f \tau_{2}\right),
$$

where the last equation applies under the assumption of having $\sigma^{2}(1)+\sigma^{2}(2)=1$ and both, $\sigma^{2}(1)$ and $\sigma^{2}(2)$, are non-zeros. From (47) it is clear that $0 \leq \rho_{x_{1}, x_{2}} \leq 1$.

\section{REFERENCES}

[1] E. Telatar, "Capacity of multi-antenna gaussian channels," European Trans. Telecommun., vol. 10, no. 6, pp. 585-595, Nov./Dec. 1999.

[2] T. M. Cover and A. E. Gamal, "Capacity theorems for the relay channel," IEEE Trans. Inform. Theory, vol. 25, no. 9, pp. 572-584, Sept. 1979.

[3] J. N. Laneman, D. N. C. Tse, and G. W. Wornell, "Cooperative diversity in wireless networks: Efficient protocols and outage behavior," IEEE Trans. Inform. Theory, vol. 50, no. 12, pp. 3062-3080, Dec. 2004.

[4] J. N. Laneman and G. W. Wornell, "Distributed space-time coded protocols for exploiting cooperative diversity in wireless networks," IEEE Trans. Inform. Theory, vol. 49, no. 10, pp. 2415-2425, Oct. 2003.

[5] M. Dohler, B. A. Rassool, and A. H. Aghvami, "Performance evaluation of sttcs for virtual antenna arrays," in Proc. IEEE VTC Spring 2003, vol. 1, pp. 57-60, Apr. 2003.

[6] P. A. Anghel, G. Leus, and M. Kaveh, "Multi-user space-time coding in cooperative networks," in Proc. International Conference on Acoustics, Speech and Signal Processing (ICASSP), Apr. 6-10 2003.

[7] K. G. Seddik, A. K. Sadek, A. S. Ibrahim, and K. J. R. Liu, "Design criteria and performance analysis for distributed space-time coding," to appear in IEEE Trans. Veh. Technol., 2008.

[8] W. Su, Z. Safar, and K. J. R. Liu, "Full-rate full-diversity spacefrequency codes with optimum coding advantage," IEEE Trans. Inform. Theory, vol. 51, no. 1, pp. 229-249, Jan. 2005.

[9] Y. Li, W. Zhang, and X.-G. Xia, "Distributive high-rate full-diversity space-frequency codes achieving full cooperative and multipath diversity for asynchronous cooperative communications," in Proc. IEEE Global Communications Conference (GLOBECOM 2006), Dec. 2006.

[10] P. Merkey and E. C. Posner, "Optimal cyclic redundancy codes for noise channels," IEEE Trans. Inform. Theory, vol. 30, pp. 865-867, Nov. 1984.

[11] A. K. Sadek, W. Su, and K. J. R. Liu, "Multi-node cooperative communications in wireless networks," IEEE Trans. Signal Processing, vol. 55, pp. 341-355, Jan. 2007.

[12] S. Siwamogsatham, M. P. Fitz, and J. H. Grimm, "A new view of performance analysis of transmit diversity schemes in correlated rayleigh fading," IEEE Trans. Inform. Theory, vol. 48, no. 4, pp. 950-956, Apr. 2002.

[13] R. A. Horn and C. R. Johnson, Topics in Matrix Analysis. Cambridge Univ. Press, 1991.

[14] H. L. V. Trees, Detection, Estimation, and Modulation Theory-Part (I). Wiley, 1968.

[15] M. O. Hasna and M. S. Alouini, "End-to-end performance of transmission systems with relays over rayleigh fading channels," IEEE Trans. Wireless Commun., vol. 2, pp. 1126-1131, Nov. 2003.

[16] H. Holm and M. S. Alouini, "Sum and difference of two squared correlated nakagami variates in connection with the mckay distribution," IEEE Trans. Commun., vol. 52, no. 8, pp. 1367-1376, Aug. 2004.

[17] I. S. Gradshteyn and I. M. Ryshik, Table of Integrals, Series and Products, 6th. ed. Academic Press, 2000.

[18] R. K. Mallik, "On multivariate rayleigh and exponential distributions," IEEE Trans. Inform. Theory, vol. 49, no. 6, pp. 1499-1515, June 2003.

[19] X. Giraud, E. Boutillon, and J. C. Belfiore, "Algebraic tools to build modulation schemes for fading channels," IEEE Trans. Inform. Theory, vol. 43, no. 3, pp. 938-952, May 1997.

[20] J. Boutros and E. Viterbo, "Signal space diversity: a power- and bandwidth-efficient diversity technique for the rayleigh fading channel," IEEE Trans. Inform. Theory, vol. 44, no. 4, pp. 1453-1467, July 1998. 


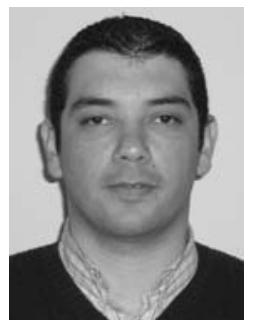

Karim G. Seddik ( $\mathrm{S}^{\prime} 04$ ) is an assistant professor in the Electrical Engineering Department at Alexandria University, Egypt. Dr. Seddik received the B.S. (with highest honors) and M.S. degrees in electrical engineering from Alexandria University, Alexandria, Egypt, in 2001 and 2004, respectively. He received his Ph.D. degree at the Electrical and Computer Engineering Department, University of Maryland, College Park 2008. His research interests include cooperative communications and networking, MIMOOFDM systems, and distributed detection in wireless sensor networks. Dr. Seddik is a recipient of the Certificate of Honor from the Egyptian President for being ranked first among all departments in College of Engineering, Alexandria University in 2002, the Graduate School Fellowship from the University of Maryland in 2004 and 2005 and the Future Faculty Program Fellowship from the University of Maryland in 2007.

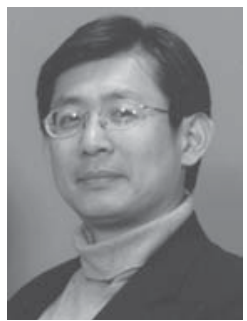

K. J. Ray Liu (F'03) is Professor and Associate Chair, Graduate Studies and Research, of Electrical and Computer Engineering Department, and Distinguished Scholar-Teacher of University of Maryland, College Park. He leads the Maryland Signals and Information Group conducting research encompassing broad aspects of information technology including signal processing, communications, networking, information forensics and security, biomedical and bioinformatics.

Dr. Liu is the recipient of numerous honors and awards including best paper awards from IEEE Signal Processing Society (twice), IEEE Vehicular Technology Society, and EURASIP; IEEE Signal Processing Society Distinguished Lecturer, EURASIP Meritorious Service Award, and National Science Foundation Young Investigator Award. He also received various teaching and research recognitions from University of Maryland including university-level Invention of the Year Award and college-level Poole and Kent Company Senior Faculty Teaching Award, and Outstanding Faculty Research Award.

Dr. Liu is Vice President - Publications and on the Board of Governor of IEEE Signal Processing Society. He was the Editor-in-Chief of IEEE SIGNAL PROCESSING MAGAZINE and the founding Editor-in-Chief of EURASIP JouRnAl on APPLIED Signal PROCESSING.

His recent books include Cooperative Communications and Networking (Cambridge University Press, 2008); Resource Allocation for Wireless Networks: Basics, Techniques, and Applications (Cambridge University Press, 2008); Ultra-Wideband Communication Systems: The Multiband OFDM Approach (IEEE-Wiley, 2007); Network-Aware Security for Group Communications (Springer, 2007); Multimedia Fingerprinting Forensics for Traitor Tracing (Hindawi, 2005). 